\title{
CROSS SECTIONALLY CONNECTED 2-SPHERES ARE TAME
}

\author{
BY R. A. JENSEN ${ }^{1}$
}

Communicated by Steve Armentrout, March 5, 1970

W. T. Eaton [4] and Norman Hosay [5] have independently shown that a 2-sphere $S$ in $E^{3}$ is tame if each horizontal cross section of $S$ is either a simple closed curve or a point. The purpose of this note is to indicate how to extend Hosay's argument to show that $S$ is tame if each horizontal cross section is connected. This answers a question raised by Bing [2].

The author would like to thank L. D. Loveland for helpful suggestions.

The notation used here is as in [5]. Let $E_{t}=\left\{(x, y, z) \in E^{3} \mid z=t\right\}$.

THEOREM. Let $S$ be a 2-sphere in $E^{3}$ such that $S \cap E_{t}$ is connected (or void) for each $t$ in $E^{1}$. Then $S$ is tame.

Let $J_{t}=S \cap E_{t}$. We suppose $\left\{t \mid J_{t} \neq \varnothing\right\}=[0,1]$. The first four parts of Hosay's proof are concerned with showing that $S$ is locally tame modulo $J_{0} \cup J_{1}$ by showing that the complementary domains of $S$ are locally simply connected at each point $p$ of $S-\left(J_{0} \cup J_{1}\right)$. For a round open ball $U$ containing $p$ he picks a certain map $h$ taking a disk $D$ into $U \cap \mathrm{Cl}($ Int $S$ ) and wishes to construct a map $g: D \rightarrow U-S$ which agrees with $h$ on $\mathrm{Bd} D$.

We first observe that since a separable metric space can contain only countably many mutually disjoint separators which are not irreducible, the set $J_{t}, 0<t<1$, is an irreducible separator of $S$ (and hence of $E_{t}$ ) except for at most countably many values of $t$. Using Cannon's result [3] we know that each set $J_{t}, 0<t<1$, is a taming set. We next observe that if $\left\{J_{i}\right\}$ is a countable collection of taming sets on $S$ the techniques of [1] can be used to construct an $\epsilon$-map of $\mathrm{Cl}\left(\right.$ Int $S$ ) into $\mathrm{Cl}(\mathrm{Int} S)-\cup J_{i}$. (Proofs of these observations appear in [6].) Thus we may suppose that $h(D) \cap J_{t}=\varnothing$ unless $J_{t}$ is an irreducible separator of $E_{t}$. This is the key to extending Hosay's argument.

In part (A) of [5] Hosay uses the fact that if $h\left(A_{t}^{t}\right)$ is a certain continuum in $h(D) \cap E_{t}$ then any two points of $h\left(A_{i}^{t}\right) \cap \operatorname{Int} S$ can be

AMS 1969 subject classifications. Primary 5705; Secondary 5478.

Key words and phrases. Tame 2 -spheres, tame surfaces, surfaces in $E^{3}$.

1 The results presented in this paper are a part of the author's dissertation at the University of Wisconsin, written under the direction of R. H. Bing. 
joined by an arc in $E_{t} \cap U \cap$ Int $S$. But this can still be done under our weaker hypotheses, since either $h\left(A_{i}^{t}\right) \subset$ Int $S$ or else $J_{t}$ is an irreducible separator of $E_{t}$ and we can apply the lemma at the end of this paper, letting $E_{t} \cap$ Int $S$ be $G, h\left(A_{i}^{t}\right)$ be $C$, and $E_{t} \cap U$ be $N$.

In part (B) we need to know that each component of $E_{t} \cap \operatorname{Int} S \cap U$ is simply-connected. But this will always be the case as long as $U$ is chosen small enough so that it does not contain $J_{t}$ for any $t$ in $[0,1]$.

The rest of Hosay's proof that Int $S$ is locally simply connected at $p$ can be used without comment.

To show that $S$ is also locally tame at each point of $J_{0}$ and $J_{1}$ we note first that if $J_{0}$, for example, is nondegenerate then it is a taming set [3]. Thus $S$ would be locally tame at each point of $J_{0}$. If $J_{0}$ is a point it is not hard to construct a tame arc piercing $S$ at $J_{0}$. (Details are given in [6].) Thus $S$ would still be locally tame at $J_{0}$. Similarly, $S$ is locally tame at each point of $J_{1}$. Thus $S$ is tame.

We are finished when we prove the following lemma used above to enable part (A) of Hosay's construction to be carried out.

Lemma. Suppose $G$ is a complementary domain of an irreducible separator of the plane, and suppose $C$ is a compact continuum in $\mathrm{Cl}(G)$. Then if $N$ is any planar neighborhood of $C$ each pair of points in $C \cap G$ can be joined by an arc in $G \cap N$.

Proof. Let $p$ and $q$ be two points of $C \cap G$, and let $J$ be the irreducible separator of the plane.

In general we may suppose that $N$ is the interior of a disk with holes and $\mathrm{Bd} N$ is a finite collection of simple closed curves. The lemma follows when we show that $\mathrm{Bd} N \cap G$ can not separate $p$ from $q$ in $G$.

From the unicoherence of the open disk we may conclude that if Bd $N \cap G$ separates $p$ from $q$ in $G$, then some component of Bd $N \cap G$ separates $p$ from $q$ in $G$. Suppose such is the case and call this component $\alpha$. We will arrive at a contradiction.

Since $\mathrm{Cl}(\alpha) \subset \mathrm{Bd} N$ we know $\mathrm{Cl}(\alpha)$ does not separate $p$ from $q$ in the plane, $E^{2}$. Since $E^{2}-G$ does not separate them either we know from Janiszewski's Theorem that $\left(E^{2}-G\right) \cap \mathrm{Cl}(\alpha)$ is neither connected nor void. Thus $\mathrm{Cl}(\alpha)-\alpha$ contains at least two points, and hence $\alpha$ is the interior of an $\operatorname{arc}$ on $\mathrm{Bd} N$, whose endpoints we call $x$ and $y$.

Let $\gamma$ be an arc in $G$ from $p$ to $q$ which intersects $\alpha$ in exactly one point, where it pierces $\alpha$. Pick connected neighborhoods $N_{x}$ and $N_{y}$ of $x$ and $y$ respectively which do intersect $\gamma$ or $C$. Let $G^{\prime}$ be a complementary domain of $J$ different from $G$. Since $J$ is an irreducible separator $x$ and $y$ must be in $\mathrm{Cl}\left(G^{\prime}\right)$. Thus $G^{\prime}$ intersects $N_{x}$ and $N_{y}$. Hence 
there is a simple closed curve $K$ in $\alpha \cup N_{x} \cup G^{\prime} \cup N_{y}$ which contains $\alpha \cap \gamma$. But now $K$ must separate $p$ from $q$ since $\gamma$ pierces it, yet the continuum $C$ contains $p$ and $q$ and misses $K$. Thus the assumption that $\mathrm{Bd} N \cap G$ separates $p$ from $q$ in $G$ leads to a contradiction.

\section{REFERENCES}

1. R. H. Bing, Pushing a 2-sphere into its complement, Michigan Math. J. 11 (1964), 33-45. MR 28 \#3408.

2. - Topology Seminar (Wisconsin, 1965) Ann. of Math. Studies, no. 60, Princeton Univ. Press, Princeton, N.J., 1966, p. 82. MR 34 \#1974.

3. J. W. Cannon, Characterization of taming sets on 2-spheres, Notices Amer. Math. Soc. 15 (1968), 768. Abstract \#658-163.

4. W. T. Eaton, Cross sectionally simple spheres, Bull. Amer. Math. Soc. 75 (1969), 375-378. MR 39 \#957.

5. Norman Hosay, $A$ proof of the slicing theorem for 2-spheres, Bull. Amer. Math. Soc. 75 (1969), 370-374. MR 39 \#956.

6. R. A. Jensen, Cross sectionally connected spheres, Thesis, Univ. of Wisconsin, Madison, Wis., 1969.

University of Wisconsin, Madison, Wisconsin 53706

University of Miami, Coral Gables, Florida 33124 